\title{
Uncertainty in Basic Short-Term Macroeconomic Models with Angel-Daemon Games
}

\section{Joaquim Gabarro}

Computer Science Dept. ALBCOM Research Group,

Universitat Politècnica de Catalunya (UPC), BarcelonaTech,

Barcelona, Spain

E-mail: gabarro@cs.upc.edu

\section{Maria Serna}

Computer Science Dept. ALBCOM Research Group, Universitat Politècnica de Catalunya (UPC), BarcelonaTech, Barcelona, Spain

E-mail: mjserna@cs.upc.edu

\begin{abstract}
We propose the use of an angel-daemon framework to perform an uncertainty analysis of short-term macroeconomic models. The angel-daemon framework defines a strategic game where two agents, the angel and the daemon, act selfishly. These games are defined over an uncertainty profile which presents a short and macroscopic description of a perturbed situation. The Nash equilibria on these games provide stable strategies in perturbed situations, giving a natural estimation of uncertainty. We apply the framework to the the uncertainty analysis of linear versions of the IS-LM and the IS-MP models.
\end{abstract}

Keywords: Uncertainty profiles; strategic games; zero-sum games; angeldaemon games; IS-LM model; IS-MP model

Biographical notes: Joaquim Gabarro studied Physics and Computer Science, obtained his Ph.D. at UPC in 1983. From 1985 he is Associate Professor at UPC. He has co-authored over 70 scientific contributions published at international journals, conference proceedings and book chapters.

Maria Serna obtained her Ph.D. at UPC in 1990. She is currently Full Professor at the Computer Science Department of (UPC) since 2006. She has co-authored over 100 scientific contributions published at international journals, conference proceedings as well as book chapters.

\section{Introduction}

The distinction between risk and uncertainty has become increasingly important since (Knight, 1921) discussed it as we have imperfect knowledge of future events in our everchanging world. Informally, risk can be measured by probabilities. In contrast, uncertainty refers to something where we cannot even gather the information required to figure out probabilities. However, in practice there is no difference between risk and uncertainty in empirical analysis on the economy and financial markets. Both are measured by historical 
standard deviation of the variable of interest (Hull, 2010; Arratia, 2014). This paper proposes an alternative to disentangle these seemingly indistinguishable concepts applying ideas from game theory and computer science.

The study of web applications is a field where uncertainty becomes unavoidable. The angel-daemon framework (Gabarro et al., 2014) provides a way to obtain numerical estimates of uncertainty in the execution of a Web service. Reality often evolves in between optimism and pessimism. To model this situation, the framework considers two agents : the angel $(\mathfrak{a})$, dealing with the optimistic side; and the daemon $(\mathfrak{d})$, dealing with the pessimistic side. These agents act strategically in an associated angel-daemon game. Here, we present an angel-daemon $(\mathfrak{a} / \mathfrak{d})$ framework to model uncertainty in short-term macroeconomic models. In the $\mathfrak{a} / \mathfrak{d}$ approach the actions undertaken by $\mathfrak{a}$ and $\mathfrak{d}$ usually go into different directions and can affect the result of underlying systems in unexpected ways. In some cases, $\mathfrak{a}$ or $\mathfrak{d}$ may be identified with policy makers or institutions. In other cases, they may describe a situation created by many interacting agents. To test the applicability of the $\mathfrak{a} / \mathfrak{d}$ framework, we start with linear approximation of extensively studied models. In particular, we develop $\mathfrak{a} / \mathfrak{d}$ analysis of the InvestmentSavings-LiquidityMoney (IS-LM) introduced by (Hicks, 1937, 1980-1981) and the InvestmentSavings-MonetaryPolicy (IS-MP) developped by (Romer, 2000).

In order to make this paper self contained for the wide audience, we describe the two basic Macroeconomic models considered in this paper. The InvestmentSavingsLiquidityMoney model (IS-LM) (Hicks, 1937) provides a way to express, in equilibrium, the national income and the interest rate as a a function of several exogenous components. Despite its simplicity, this model continues making useful predictions (Krugman, 2011). The IS line $Y=C(Y-T)+I(r)+G$ represents a continuum of equilibria in the goods market. On it, $Y$ is the national income, $r$ is the interest rate. The remaining components are the sum of the annual rates of spending by: the consumers (as a function of the disposable income) $C(Y-T)$; the investors (as a function of the interest rate) $I(r)$ and the government $G$. The $\mathrm{LM}$ line $M / P=L(r, Y)$ is interpreted as continuum of equilibrium in the money market. The money supply is $M / P$, where $M$ is the money and $P$ is the price level. The liquidity preference $L(r, Y)$ is a function of the national income and the interest rate. An equilibrium point $(Y, r)$ is a solution of the system of equations. These equilibria correspond to the points where both markets are at mutual equilibrium.

In the thirties, the world was in transition from the gold standard and the monetary policy of the central banks changed to deal with inflation. The Central Bank creates new money by lending to banks for very short periods (Piketty, 2014). The primary objective of the European Central Bank is to maintain price stability. The European Central Bank aims at maintaining inflation rates below, but close to, $2 \%$ over the medium term (ECB, 2015). The InvestementSaving-MonetaryPolicy model (IS-MP) (Romer, 2000) considers this reality. We start with the dynamic aggregate demand/aggregate supply model, (Mankiw, 2013). The supply for goods and services is given by $Y_{t}=\bar{Y}_{t}-\alpha\left(r_{t}-\rho\right)+\epsilon_{t}$. In this equation, the total output for goods and services is $Y_{t}$ and the economy's natural output is $\bar{Y}_{t}$. The parameter $\alpha>0$ measures the sensitivity of the demand in front of the real interest rate $r_{t}$ and $\rho$ is the natural rate of interest. The parameter $\epsilon_{t}$ represents the random demand shock. The real interest rate $r_{t}$ is given by a simplified version of Fisher's equation with no expectations: $r_{t}=i_{t}-\pi_{t}$ where $i_{t}$ is the nominal interest rate and $\pi_{t}$ is the inflation rate. The inflation $\pi_{t}$ at period $t$ is described by a version of the Phillips curve as a function of the past inflation $\pi_{t-1}$ and with no expectations: $\pi_{t}=\pi_{t-1}+\phi\left(Y_{t}-\bar{Y}_{t}\right)+v_{t}$. The parameter $\phi>0$ measures the responsiveness of the inflation to output fluctuations and 
Uncertainty in Basic Macroeconomic models

\begin{tabular}{||l|c||}
\hline Variables & $\mathcal{V}$ \\
\hline Taxes & $0<T$ \\
Exogenous government spending & $0<G$ \\
Money Supply & $0<M$ \\
Price index & $0<P$ \\
\hline Parameters & $\mathcal{P}$ \\
\hline Autonomous consumption & $0<a$ \\
Marginal propensity to consume & $0<b<1$ \\
Exogenous investment & $0<c$ \\
Interest sensitivity & $0<d$ \\
Income sensitivity for real money & $0<e$ \\
Interest sensitivity for real money & $0<f$ \\
\hline
\end{tabular}

Figure 1 The exogenous components in the linear approximation to the IS-LM model.

$v_{t}$ is the random supply shock. The monetary policy is based on Taylor's Rule (Taylor, 1993). The nominal interest rate $i_{t}$ is given by $i_{t}=\pi_{t}+\rho+\theta_{\pi}\left(\pi_{t}-\pi_{t}^{*}\right)+\theta_{Y}\left(Y_{t}-\bar{Y}_{t}\right)$. In this equation, $\pi_{t}^{*}$ is the central bank's target inflation rate and $\theta_{\pi}>0, \theta_{Y}>0$ measure responsiveness. We avoid temporal dependencies and we consider a version of the IS-MP model assuming that past inflation coincides with the Central Bank target inflation. As before an equilibrium point $(Y, \pi)$ is a solution of the system of equations.

The paper is structured as follows. Section 2 is devoted to the linear approximations and exogenous components. In Section 3, we provide a model for perturbations of the exogenous components, the so called perturbation strength model. Section 4 introduces uncertainty profiles and the associated $\mathfrak{a} / \mathfrak{d}$ games tailored to the linear IS-LM and IS-MP models and analyses the Nash equilibria of some cases. Section 5 studies the IS-LM model when we are uncertain of the fiscal policy and Section 6 studies the IS-MP model when we are uncertain of the external shocks. Finally, in Section 7, we raise some remarks and future research.

\section{Linear Approximations and Exogenous Components}

We use $\mathfrak{M}$ to denote a linear approximation of a model, $\mathfrak{M} \in\{$ IS-LM, IS-MP $\}$. For a model $\mathfrak{M}, \mathcal{P}_{\mathfrak{M}}$ denotes the set of exogenous parameters, $\mathcal{V}_{\mathfrak{M}}$ denotes the the set of exogenous variables and $\mathcal{E}_{\mathfrak{M}}=\mathcal{P}_{\mathfrak{M}} \cup \mathcal{V}_{\mathfrak{M}}$ is the set of exogenous components. We use set notation like $b \in \mathcal{P}_{\mathfrak{M}}$ or $T \in \mathcal{V}_{\mathfrak{M}}$. When $\mathfrak{M}$ is clear from the context we use $\mathcal{E}, \mathcal{P}$ and $\mathcal{V}$. Let us start with the linear approximation of the IS-LM model (Baldani et al., 2007).

Definition 1: The IS-LM model is described by the following equations: $C(Y-T)=$ $a+b(Y-T), I(r)=c-d r, L(r, Y)=e Y-f r$. The set exogenous components is $\mathcal{E}=$ $\mathcal{V} \cup \mathcal{P}$ with $\mathcal{V}=\{a, b, c, d, e, f\}$ and $\mathcal{P}=\{T, G, M, P\}$ (see Figure 1). 
Let us express the endogenous variables $\{Y, r\}$ in equilibrium as a function of $\mathcal{E}$. The equilibrium condition $Y=Y(r)$ (IS line) gives the equation $Y=a+b(Y-T)+c-$ $d r+G$. The condition $r=r(Y)$ (MP line) gives $M / P=e Y-f r$. Thus, we get

$$
Y=\frac{1}{(1-b)}(a+c+G-b T-d r) \text { and } r=\frac{1}{f}\left(e Y-\frac{M}{P}\right) .
$$

With matrices:

$$
\left(\begin{array}{cc}
1-b & d \\
P e & -P f
\end{array}\right)\left(\begin{array}{l}
Y \\
r
\end{array}\right)=\left(\begin{array}{c}
a+c+G-b T \\
M
\end{array}\right) .
$$

Solving the system, we get $(Y, r)$.

$$
\left(\begin{array}{l}
Y \\
r
\end{array}\right)=\frac{1}{(1-b) f+d e}\left(\begin{array}{cc}
f & d / P \\
e & -(1-b) / P
\end{array}\right)\left(\begin{array}{c}
a+c+G-b T \\
M
\end{array}\right) .
$$

and defining $g=(1-b) f+d e$ we get:

$$
Y=\frac{f}{g}(a+c+G-b T)+\frac{d}{g} \frac{M}{P} \text { and } r=\frac{e}{g}(a+c+G-b T)-\frac{(1-b)}{g} \frac{M}{P} .
$$

As $(Y, r)$ depends on $\mathcal{E}$, when needed we write $(Y(\mathcal{E}), r(\mathcal{E}))$.

Example 2.1: Consider the following $\mathcal{E}$ :

\begin{tabular}{||c|c|c|c|c|c|c|c|c|c||}
\hline$a$ & $b$ & $c$ & $d$ & $e$ & $f$ & $T$ & $G$ & $M$ & $P$ \\
\hline 200 & $3 / 4$ & 200 & 25 & 1 & 100 & 100 & 100 & 1000 & 2 \\
\hline
\end{tabular}

The linear system is $Y=1700-100 r, r=Y / 100-5$, solving $Y=1100, r=6$.

We present now the linear approximation to the IS-MP model from (Mankiw, 2013).

$$
\begin{aligned}
& Y_{t}=\bar{Y}_{t}-\hat{\alpha}\left(\pi_{t}-\pi_{t}^{*}\right)+\hat{\beta} \epsilon_{t} \text { where } \hat{\alpha}=\frac{\alpha \theta_{\pi}}{1+\alpha \theta_{Y}} \text { and } \hat{\beta}=\frac{1}{1+\alpha \theta_{Y}} \\
& \pi_{t}=\pi_{t-1}+\phi\left(Y_{t}-\bar{Y}_{t}\right)+v_{t} .
\end{aligned}
$$

We consider a simplified linear approximation in which we avoid dependences of inflation on their lagged values. We consider only the case where the past inflation coincides with the Central Bank target inflation, i.e. $\pi_{t-1}=\pi_{t}^{*}$. Furthermore, assuming that period $t$ is known, we drop the sub-index.

Definition 2: The IS-MP model is described by the equations: $Y=\bar{Y}-\hat{\alpha}\left(\pi-\pi^{*}\right)+\hat{\beta} \epsilon$ and $\pi=\pi^{*}+\phi(Y-\bar{Y})+v$. The set of exogenous components $\mathcal{E}=\mathcal{V} \cup \mathcal{P}$ is given by $\mathcal{V}=\left\{\pi^{*}, \bar{Y}, \epsilon, v\right\}$ and $\mathcal{P}=\left\{\alpha, \rho, \phi, \theta_{\pi}, \theta_{Y}\right\}$ (see Figure 2). 


\begin{tabular}{||l|c||}
\hline Variables & $\mathcal{V}$ \\
\hline Central bank's target inflation & $\pi^{*}$ \\
Natural level of output & $\bar{Y}$ \\
Shock to $Y$ & $\epsilon$ \\
Shock to $\pi$ & $v$ \\
\hline Parameters & $\mathcal{P}$ \\
\hline$Y$ sensitivity to $r$ & $0<\alpha$ \\
Natural interest rate & $0<\rho$ \\
$\pi$ sensitivity to $Y$ in Philips line & $0<\phi$ \\
$i$ sensitivity to inflation in MP & $0<\theta_{\pi}$ \\
$i$ sensitivity to $Y$ in MP & $0<\theta_{Y}$ \\
\hline
\end{tabular}

Figure 2 The exogenous components in the linear approximation to the IS-MP model.

Solving the system, $Y=\bar{Y}+\hat{\gamma} \epsilon-\hat{\delta} v$ and $\pi=\pi^{*}+\hat{\rho} \epsilon+\hat{\mu} v$ where

$$
\begin{aligned}
& \hat{\gamma}=\frac{\hat{\beta}}{1+\hat{\alpha} \phi}=\frac{1}{1+\alpha\left(\theta_{Y}+\phi \theta_{\pi}\right)} \text { and } \hat{\delta}=\frac{\hat{\alpha}}{1+\hat{\alpha} \phi}=\frac{\alpha \theta_{\pi}}{1+\alpha\left(\theta_{Y}+\phi \theta_{\pi}\right)} \\
& \hat{\rho}=\frac{\phi \hat{\beta}}{1+\hat{\alpha} \phi}=\frac{\phi}{1+\alpha\left(\theta_{Y}+\phi \theta_{\pi}\right)} \text { and } \hat{\mu}=\frac{1}{1+\hat{\alpha} \phi}=\frac{1+\alpha \theta_{Y}}{1+\alpha\left(\theta_{Y}+\phi \theta_{\pi}\right)} .
\end{aligned}
$$

In matrix form:

$$
\begin{aligned}
\left(\begin{array}{l}
Y \\
\pi
\end{array}\right) & =\left(\begin{array}{rr}
\hat{\gamma} & -\hat{\delta} \\
\hat{\rho} & \hat{\mu}
\end{array}\right)\left(\begin{array}{l}
\epsilon \\
v
\end{array}\right)+\left(\begin{array}{c}
\bar{Y} \\
\pi^{*}
\end{array}\right) \\
\left(\begin{array}{l}
Y \\
\pi
\end{array}\right) & =\frac{1}{1+\alpha\left(\theta_{Y}+\phi \theta_{\pi}\right)}\left(\begin{array}{rr}
1 & -\alpha \theta_{\pi} \\
\phi & 1+\alpha \theta_{Y}
\end{array}\right)\left(\begin{array}{l}
\epsilon \\
v
\end{array}\right)+\left(\begin{array}{c}
\bar{Y} \\
\pi^{*}
\end{array}\right) .
\end{aligned}
$$

The equilibrium point $(Y, \pi)$ is a function of $\mathcal{E}$, when needed we write $(Y(\mathcal{E}), \pi(\mathcal{E}))$.

Example 2.2: Consider the following $\mathcal{E}$ for the IS-MP model:

\begin{tabular}{||c|c|c|c|c|c|c|c|c||}
\hline$\alpha$ & $\rho$ & $\phi$ & $\theta_{\pi}$ & $\theta_{Y}$ & $\pi^{*}$ & $\bar{Y}$ & $\epsilon$ & $v$ \\
\hline 1 & 2 & $1 / 4$ & $1 / 2$ & $1 / 2$ & 2 & 100 & 1 & $1 / 2$ \\
\hline
\end{tabular}

We can compute directly

$$
\left(\begin{array}{l}
Y \\
\pi
\end{array}\right)=\frac{1}{13}\left(\begin{array}{cc}
8 & -4 \\
2 & 12
\end{array}\right)\left(\begin{array}{c}
1 \\
1 / 2
\end{array}\right)+\left(\begin{array}{c}
100 \\
2
\end{array}\right)=\frac{1}{13}\left(\begin{array}{c}
1306 \\
34
\end{array}\right)
$$

Therefore $Y=1306 / 13$ and $\pi=34 / 13$. 


\section{Perturbation Strength Model}

We start with a description of the perceived potential perturbations in the model.

Definition 3: Let $\mathfrak{M}$ be a macroeconomic model and let $\mathcal{E}$ be the set of its exogenous components. A perturbation strength model for $\mathcal{E}$ is a set of $\mathcal{S}$ of pairs of real numbers, i.e., $\mathcal{S}=\left\{\left(\delta_{\mathfrak{a}}(e), \delta_{\mathfrak{d}}(e)\right) \mid e \in \mathcal{E}\right\}$ describing the potential changes that can be applied to the valuations of the exogenous components by $\mathfrak{a}$ and $\mathfrak{d}$.

Observe that perturbations are real numbers, so they can be either positive or negative.

Example 3.1: A perturbation strength model $\mathcal{S}$ for the IS-LM model $\mathcal{E}$ given in Example 2.1 is:

\begin{tabular}{||c||c|c|c||c|c|c|c||}
\hline agent & $a$ & $b$ & $c, d, e, f$ & $T$ & $G$ & $M$ & $P$ \\
\hline $\mathfrak{a}$ & 0 & $+1 / 20$ & 0 & 0 & +50 & 0 & 0 \\
$\mathfrak{d}$ & 0 & 0 & 0 & +50 & -25 & 0 & +1 \\
\hline
\end{tabular}

The angel $\mathfrak{a}$ has the ability to act upon the parameters $\{b, G\}$, then $\delta_{\mathfrak{a}}(b)=1 / 20, \delta_{\mathfrak{a}}(G)=$ 50 an For any $e \in \mathcal{E} \backslash\{b, G\}, \mathfrak{a}, \delta_{\mathfrak{a}}(e)=0$. The daemon $\mathfrak{d}$ has the ability to act upon some of the parameters in $\{P, T, G\}$, then $\delta_{\mathfrak{d}}(P)=1, \delta_{\mathfrak{d}}(T)=50, \delta_{\mathfrak{d}}(G)=-25$ and for $e \in \mathcal{E} \backslash\{P, T, G\}, \delta_{\mathfrak{d}}(e)=0$.

Example 3.2: In this example $\mathfrak{a}$ and $\mathfrak{d}$ can perturb the predetermined variables $\mathcal{V}$ in the IS-MP model given in Example 2.2. One perturbation strength model $\mathcal{S}$ is

\begin{tabular}{||c||c||c|c|c|c||}
\hline agent & $\alpha, \rho, \phi, \theta_{\pi}, \theta_{Y}$ & $\pi^{*}$ & $\bar{Y}$ & $\epsilon$ & $v$ \\
\hline $\mathfrak{a}$ & 0 & 0 & +25 & +2 & 0 \\
$\mathfrak{d}$ & 0 & +3 & 0 & 0 & +2 \\
\hline
\end{tabular}

In $\mathcal{S}, \mathfrak{a}$ can potentially act over $\bar{Y}$ and $\epsilon$. That is $\delta_{\mathfrak{a}}(\bar{Y})=25$ and $\delta_{\mathfrak{a}}(\epsilon)=2$ but, for any other $e \in\left\{\alpha, \rho, \phi, \theta_{\pi}, \theta_{Y}, \pi^{*}, v\right\}, \delta_{\mathfrak{a}}(e)=0 . \mathfrak{d}$ can potentially act over $\pi^{*}$ and $v$ with $\delta_{\mathfrak{d}}\left(\pi^{*}\right)=3$ and $\delta_{\mathfrak{d}}(v)=2$, all other $\delta_{\mathfrak{d}}(e)=0$.

For a set $s \subseteq \mathcal{E}, \# s$ denotes the number of components in $s$.

Definition 4: Consider a model $\mathfrak{M}$ having exogenous components $\mathcal{E}$ under a perturbation strength model $\mathcal{S}$. Given a joint action $(a, d)$ with $a, d \subseteq \mathcal{E}$. The valuation under strength $\mathcal{E}^{\prime}$ of $\mathcal{E}$ given $\mathcal{S}$ and $(a, d)$ is noted strength ${ }_{\mathcal{S}}(\mathcal{E})[a, d]$ and it is defined as follows. For any $e \in \mathcal{E}$, strength $_{\mathcal{S}}(e)[a, d]=e+\delta_{\mathcal{S}}(e)[a, d]$ where

$$
\delta_{\mathcal{S}}(e)[a, d]= \begin{cases}0 & e \notin a \cup d \\ \delta_{\mathfrak{a}}(e) & e \in a \backslash d \\ \delta_{\mathfrak{d}}(e) & e \in d \backslash a \\ \delta_{\mathfrak{a}}(e)+\delta_{\mathfrak{d}}(e) & e \in a \cap d\end{cases}
$$

finally, $\operatorname{strength}_{\mathcal{S}}(\mathcal{E})[a, d]=\left\{\operatorname{strength}_{\mathcal{S}}(e)[a, d] \mid e \in \mathcal{E}\right\}$ 
Let us move to the computation of the equilibrium point in the valuation obtained after a joint action $(a, d)$ of the two agents. We denote the equilibrium point as $\left(Y\right.$ strength $\left._{\mathcal{S}}(\mathcal{E})[a, d]\right), r\left(\right.$ strength $\left._{\mathcal{S}}(\mathcal{E})[a, d]\right)$. When $\mathcal{S}$ is clear from the context we note strength $_{\mathcal{S}}(\mathcal{E})[a, d]$ as $\mathcal{E}(a, d)$ and the equilibrium point as $(Y(a, d), r(a, d))$. The following result points out some basic properties (proof straightforward).

Lemma 1: Let $\mathfrak{M}$ be a model having exogenous components $\mathcal{E}$ under a perturbation strength model $\mathcal{S}$ and consider a joint action $(a, d)$. Then strength ${ }_{\mathcal{S}}(e)[a, d]=e$ if and only if either $e \notin a \cup d$ or $\delta_{\mathfrak{a}}(e)=\delta_{\mathfrak{d}}(e)=0$. The whole system remains unperturbed, i.e. strength $_{\mathcal{S}}(\mathcal{E})[a, d]=\mathcal{E}$ when $\mathcal{S}=\{(0,0) \mid e \in \mathcal{E}\}$ or $(a, d)=(\emptyset, \emptyset)$.

Following, we provide an application of the Definition 4 to the IS-LM model.

Example 3.3: We continue with Examples 2.1 and 3.1 under the joint action $(a, d)=$ $(\{b\},\{P, G\})$. After the joint action we get a new valuation, letting $\mathcal{E}^{\prime}=\mathcal{E}(\{b\},\{P, G\})$ and $e^{\prime}=\operatorname{strength}(e)[\{b\},\{P, G\}]$. We have $\mathcal{E}^{\prime}=\operatorname{strength}_{\mathcal{S}}(\mathcal{E})[\{b\},\{P, G\}]=$ $\left\{a^{\prime}, b^{\prime}, c^{\prime}, d^{\prime}, e^{\prime}, f^{\prime}, T^{\prime}, G^{\prime}, M^{\prime}, P^{\prime}\right\}$. The values of $\mathcal{E}^{\prime}$ are given in the following table.

\begin{tabular}{||l||l||c|l|l|l|l|l||c|c|c|c||}
\hline agent & choice & $a$ & $b$ & $c$ & $d$ & $e$ & $f$ & $T$ & $G$ & $M$ & $P$ \\
\hline & & 200 & $3 / 4$ & 200 & 25 & 1 & 100 & 100 & 100 & 1000 & 2 \\
\hline $\mathfrak{a}$ & $a=\{b\}$ & & $+1 / 20$ & & & & & & & & \\
$\mathfrak{d}$ & $d=\{P, G\}$ & & & & & & & & -25 & & +1 \\
\hline & & $a^{\prime}$ & $b^{\prime}$ & $c^{\prime}$ & $d^{\prime}$ & $e^{\prime}$ & $f^{\prime}$ & $T^{\prime}$ & $G^{\prime}$ & $M^{\prime}$ & $P^{\prime}$ \\
\hline & & 200 & $4 / 5$ & 200 & 25 & 1 & 100 & 100 & 75 & 1000 & 3 \\
\hline
\end{tabular}

The equilibrium point corresponding to $\mathcal{E}(\{b\},\{P, G\})$ is $Y(\{b\},\{P, G\})=28700 / 27 \approx$ 1062.96, $r(\{b\},\{P, G\})=197 / 27 \approx 7.29$.

When $\mathfrak{M}$ is perturbed from valuation $\mathcal{E}$ into $\mathcal{E}^{\prime}$ by joint action $(a, d)$, we would like to isolate the effects of the the perturbation by expressing the equilibrium point $(Y(a, d), r(a, d))$ with respect to the non-perturbed equilibrium point $(Y, r)$. The proofs of the following lemmas are straightforward.

Lemma 2: Consider a perturbation strength model $\mathcal{S}$ for the IS-LM model such that, for $e \in\{b, d, e, f, P\}$, we have $\delta_{\mathfrak{a}}(e)=\delta_{\mathfrak{d}}(e)=0$. Let $(a, d)$ be a joint action and define

$$
\delta_{\mathcal{S}}(a, c, G, T)[a, d]=\delta_{\mathcal{S}}(a)[a, d]+\delta_{\mathcal{S}}(c)[a, d]+\delta_{\mathcal{S}}(G)[a, d]-b \delta_{\mathcal{S}}(T)[a, d] .
$$

Then, it holds

$$
\left(\begin{array}{c}
Y(a, d) \\
r(a, d)
\end{array}\right)=\left(\begin{array}{c}
Y \\
r
\end{array}\right)+\frac{1}{g}\left(\begin{array}{cc}
f & d / P \\
e & -(1-b) / P
\end{array}\right)\left(\begin{array}{c}
\delta_{\mathcal{S}}(a, c, G, T)[a, d] \\
\delta_{\mathcal{S}}(M)[a, d]
\end{array}\right)
$$

We are also interested in valuations $\mathcal{E}$ in relation to fiscal policies.

Lemma 3: Consider a perturbation strength model $\mathcal{S}$ where $\{G, T\}$ are the unique components that can be perturbed. For a joint action $(a, d)$, we have

$$
\left(\begin{array}{c}
Y(a, d) \\
r(a, d)
\end{array}\right)=\left(\begin{array}{c}
Y \\
r
\end{array}\right)+\frac{1}{g} \delta_{\mathcal{S}}(G, T)[a, d]\left(\begin{array}{l}
f \\
e
\end{array}\right)
$$


where $\delta_{\mathcal{S}}(G, T)[a, d]=\delta_{\mathcal{S}}(G)[a, d]-b \delta_{\mathcal{S}}(T)[a, d]$.

Our next result provides the equilibrium point in the IS-MP model when the exogenous parameters cannot be perturbed.

Lemma 4: Consider a perturbation strength model $\mathcal{S}$ for the IS-MP model such that, for $e \in \mathcal{P}, \delta_{\mathfrak{a}}(e)=\delta_{\mathfrak{d}}(e)=0$. For any joint action $(a, d)$ it holds that

$$
\left(\begin{array}{c}
Y(a, d) \\
\pi(a, d)
\end{array}\right)=\left(\begin{array}{c}
Y \\
\pi
\end{array}\right)+\left(\begin{array}{rr}
\hat{\gamma} & -\hat{\delta} \\
\hat{\rho} & \hat{\mu}
\end{array}\right)\left(\begin{array}{l}
\delta_{\mathcal{S}}(\epsilon)[a, d] \\
\delta_{\mathcal{S}}(v)[a, d]
\end{array}\right)+\left(\begin{array}{l}
\delta_{\mathcal{S}}(\bar{Y})[a, d] \\
\delta_{\mathcal{S}}\left(\pi^{*}\right)[a, d]
\end{array}\right) .
$$

In Section 6 we will consider the case where only the income $Y$ and the inflation $\pi$ become uncertain. For such a case we have the following expression for equilibrium point.

Lemma 5: Consider a perturbation strength model $\mathcal{S}$ for the IS-MP model such that, for $e \in \mathcal{E} \backslash\left\{\bar{Y}, \pi^{*}\right\}$, we have $\delta_{\mathfrak{a}}(e)=\delta_{\mathfrak{d}}(e)=0$. For any joint action $(a, d)$, it holds $Y(a, d)=$ $Y+\delta_{\mathcal{S}}(\bar{Y})[a, d]$ and $\pi(a, d)=\pi+\delta_{\mathcal{S}}\left(\pi^{*}\right)[a, d]$.

We illustrate the computation of the equilibrium point in a perturbed scenario.

Example 3.4: Let us continue with the Example 3.2 in the IS-MP model. Consider the joint action $(a, d)=\left(\{\epsilon\},\left\{\pi^{*}, v\right\}\right)$. Writing the new values as $e^{\prime}=\operatorname{strength}(e)\left[\left(\{\epsilon\},\left\{\pi^{*}, v\right\}\right]\right.$. As usual $\mathcal{E}^{\prime}=\mathcal{E}\left(\{\epsilon\},\left\{\pi^{*}, v\right\}\right)=\left\{\alpha^{\prime}, \rho^{\prime}, \phi^{\prime}, \theta_{\pi}^{\prime}, \theta_{Y}^{\prime}, \pi^{* \prime}, \bar{Y}^{\prime}, \epsilon^{\prime}, v^{\prime}\right\}$. A sketch of the computation of the perturbed valuation is given in the following table.

\begin{tabular}{||l||l||l|l|l|l|l||c|c|c|l||}
\hline agent & choice & $\alpha$ & $\rho$ & $\phi$ & $\theta_{\pi}$ & $\theta_{Y}$ & $\pi^{*}$ & $\bar{Y}$ & $\epsilon$ & $v$ \\
\hline & & 1 & 2 & $1 / 4$ & $1 / 2$ & $1 / 2$ & 2 & 100 & 1 & $1 / 2$ \\
\hline $\mathfrak{a}$ & $\begin{array}{l}a=\{\epsilon\} \\
d=\left\{\pi^{*}, v\right\}\end{array}$ & & & & & & & & +2 & +2 \\
$\mathfrak{d}$ & & $\alpha^{\prime}$ & $\rho^{\prime}$ & $\phi^{\prime}$ & $\theta_{\pi}^{\prime}$ & $\theta_{Y}^{\prime}$ & $\pi^{* \prime}$ & $\bar{Y}^{\prime}$ & $\epsilon^{\prime}$ & $v^{\prime}$ \\
\hline & & 1 & 2 & $1 / 4$ & $1 / 2$ & $1 / 2$ & 5 & 100 & 3 & $5 / 2$ \\
\hline
\end{tabular}

As $\epsilon \in a \backslash d$, according to the Definition 4 we have

$$
\epsilon^{\prime}=\operatorname{strength}(\epsilon)\left[\left(\{\epsilon\},\left\{\pi^{*}, v\right\}\right]=\epsilon+\delta_{\mathcal{S}}\left[\{\epsilon\},\left\{\pi^{*}, v\right\}\right]=\epsilon+\delta_{\mathfrak{a}}(\epsilon)=3 .\right.
$$

As $\pi \in d \backslash a, \pi^{* \prime}=\pi+\delta_{\mathfrak{d}}\left(\pi^{*}\right)=5$. Similarly $v^{\prime}=v+\delta_{\mathfrak{d}}(v)=5 / 2$. All other values remain unchanged. In order to obtain $\left(Y^{\prime}, \pi^{* \prime}\right)=(Y(a, d), \pi(a, d))$, by using Lemma 4, we have

$$
\left(\begin{array}{l}
Y^{\prime} \\
\pi^{\prime}
\end{array}\right)=\frac{1}{13}\left(\begin{array}{c}
1306 \\
34
\end{array}\right)+\frac{1}{13}\left(\begin{array}{rr}
8 & -4 \\
2 & 12
\end{array}\right)\left(\begin{array}{l}
\delta_{\mathfrak{a}}(\epsilon) \\
\delta_{\mathfrak{d}}(v)
\end{array}\right)+\left(\begin{array}{c}
0 \\
\delta_{\mathfrak{a}}\left(\pi^{*}\right)
\end{array}\right)=\frac{1}{13}\left(\begin{array}{c}
1314 \\
101
\end{array}\right) .
$$




\section{Uncertainty Profiles and $\mathfrak{a} / \mathfrak{d}$ games}

In this section, we present the model and tools to analyse a situation under a given perturbation strength model. Uncertainty profiles Gabarro et al. (2014) are based in three components. The first one identifies the set of exogenous components that might be perturbed. The second states the limits in the number of components that can suffer perturbation. The third component quantifies (as function of the exogenous components) the benefits for the agents. In such a setting, we are uncertain about the specific subset that will suffer the perturbation.

Definition 5: Given $\mathfrak{M}, \mathcal{E}$ and a perturbation strength $\mathcal{S}$, a uncertainty profile is a tuple $\mathcal{U}=\left\langle\mathcal{E}, \mathcal{S}, \mathcal{A}, \mathcal{D}, b_{\mathfrak{a}}, b_{\mathfrak{d}}, u_{\mathfrak{a}}, u_{\mathfrak{d}}\right\rangle$ where $\mathcal{A}, \mathcal{D} \subseteq \mathcal{E}$. The spread of the perturbation $b_{\mathfrak{a}}$ and $b_{\mathfrak{d}}$, verify $b_{\mathfrak{a}} \leq \# \mathcal{A}$ and $b_{\mathfrak{d}} \leq \# \mathcal{D}$. The exerted perturbation follows from joint actions $(a, d)$ verifying $a \subseteq \mathcal{A}, d \subseteq \mathcal{D}$ with $\# a=b_{\mathfrak{a}}$ and $\# d=b_{\mathfrak{d}}$. The effects of a joint action are measured by the utility functions $u_{\mathfrak{a}}$ and $u_{\mathfrak{d}}$. Given a joint action $(a, d), u_{\mathfrak{a}}(a, d)$ measures $\mathfrak{a}$ 's gain while $u_{\mathfrak{d}}(a, d)$ measures $\mathfrak{d}$ 's gain.

The situation described by an uncertainty profile $\mathcal{U}$ is analysed by means of an associated strategic $\mathfrak{a} / \mathfrak{d}$ game. In such a game $\mathfrak{a}$ and $\mathfrak{d}$ decide their actions strategically. For basics on game theory we refer the reader to (Osborne, 2004; Osborne \& Rubinstein, 1994)).

Definition 6: Given $\mathfrak{M}, \mathcal{E}, \mathcal{S}$ and $\mathcal{U}=\left\langle\mathcal{E}, \mathcal{S}, \mathcal{A}, \mathcal{D}, b_{\mathfrak{a}}, b_{\mathfrak{d}}, u_{\mathfrak{a}}, u_{\mathfrak{d}}\right\rangle$, the associated angeldaemon strategic game (the $\mathfrak{a} / \mathfrak{d}$ game) is defined as $\Gamma(\mathcal{U})=\left\langle\{\mathfrak{a}, \mathfrak{d}\}, A_{\mathfrak{a}}, A_{\mathfrak{d}}, u_{\mathfrak{a}}, u_{\mathfrak{d}}\right\rangle$. Game $\Gamma(\mathcal{U})$ has two players $\{\mathfrak{a}, \mathfrak{d}\}$. The player's actions are $A_{\mathfrak{a}}=\left\{a \subseteq \mathcal{A} \mid \# a=b_{\mathfrak{a}}\right\}$ and $A_{\mathfrak{d}}=$ $\left\{d \subseteq \mathcal{D} \mid \# d=b_{\mathfrak{d}}\right\}$. Their utilities are $u_{\mathfrak{a}}$ and $u_{\mathfrak{d}}$.

Notice that, in an $\mathfrak{a} / \mathfrak{d}$ game the set of strategy profiles is $A_{\mathfrak{a}} \times A_{\mathfrak{d}}$. Thus strategy profiles are permissible joint actions. Recall that, a pure Nash equilibrium is a strategy profile such that neither $\mathfrak{a}$ nor $\mathfrak{d}$ can improve the situation by himself (Osborne, 2004). Formally, a strategy profile $(a, d)$ is a pure Nash equilibrium if and only if $u_{\mathfrak{a}}(a, d) \geq$ $u_{\mathfrak{a}}\left(a^{\prime}, d\right)$, for all $a^{\prime} \in A_{\mathfrak{a}}$, and $u_{\mathfrak{d}}(a, d) \geq u_{\mathfrak{d}}\left(a, d^{\prime}\right)$, for all $d^{\prime} \in A_{\mathfrak{d}}$. We note by $\operatorname{PNE}(\Gamma)$ the set of pure Nash equilibria of $\Gamma$. When $\Gamma$ is clear from the context we just write PNE. Given $d \in A_{d}$ the best response of $\mathfrak{a}$ to $\mathfrak{d}$ 's choice $d$ is the set of strategies giving to $\mathfrak{a}$ the maximum utility, i.e., $B_{\mathfrak{a}}(d)=\left\{a \mid u_{\mathfrak{a}}(a, d) \geq u_{\mathfrak{a}}\left(a^{\prime}, d\right)\right.$ for all $\left.a^{\prime} \in A_{\mathfrak{a}}\right\}$. Similarly, $B_{\mathfrak{d}}(a)=\left\{d \mid u_{\mathfrak{d}}(a, d) \geq u_{\mathfrak{d}}\left(a, d^{\prime}\right)\right.$ for all $\left.d^{\prime} \in A_{\mathfrak{d}}\right\}$. It is well known that $(a, d) \in \mathrm{PNE}$ if and only if $a \in B_{\mathfrak{a}}(d)$ and $b \in B_{\mathfrak{d}}(a)$. Formally, PNE $=\left\{(a, d) \mid a \in B_{\mathfrak{a}}(d)\right.$ and $b \in$ $\left.B_{\mathfrak{d}}(a)\right\}$. The following lemmas are straightforward. Next lemma considers two extreme cases where neither $\mathfrak{a}$ nor $\mathfrak{d}$ have freedom to make choices.

Lemma 6: Let $\mathcal{U}=\left\langle\mathcal{E}, \mathcal{S}, \mathcal{A}, \mathcal{D}, b_{\mathfrak{a}}, b_{\mathfrak{d}}, u_{\mathfrak{a}}, u_{\mathfrak{d}}\right\rangle$ be an uncertainty profile for $\mathfrak{M}$. When $b_{\mathfrak{a}}=\# \mathcal{A}$ and $b_{\mathfrak{d}}=\# \mathcal{D}$, the only PNE of $\Gamma(\mathcal{U})$ is $(\mathcal{A}, \mathcal{D})$. When $b_{\mathfrak{a}}=0$ and $b_{\mathfrak{d}}=0$, the only PNE of $\Gamma(\mathcal{U})$ is is $(\emptyset, \emptyset)$.

Our next result shows that in an equilibrium, when one of the two agents cannot act, the other agent maximizes his utility.

Lemma 7: Let $\mathcal{U}=\left\langle\mathcal{E}, \mathcal{S}, \mathcal{A}, \mathcal{D}, b_{\mathfrak{a}}, b_{\mathfrak{d}}, u_{\mathfrak{a}}, u_{\mathfrak{d}}\right\rangle$ be an uncertainty profile for $\mathfrak{M}$. When $b_{\mathfrak{d}}=0$ it holds $\operatorname{PNE}(\Gamma(\mathcal{U}))=\left\{(a, \emptyset) \mid u_{\mathfrak{a}}(a, \emptyset)=\max _{a^{\prime} \in A_{\mathfrak{a}}} u_{\mathfrak{a}}\left(a^{\prime}, \emptyset\right)\right\}$. When $b_{\mathfrak{a}}=0$ it holds $\operatorname{PNE}(\Gamma(\mathcal{U}))=\left\{(\emptyset, d) \mid u_{\mathfrak{d}}(\emptyset, d)=\max _{d^{\prime} \in A_{\mathfrak{d}}} u_{\mathfrak{d}}\left(\emptyset, d^{\prime}\right)\right\}$. 
We present examples of $\mathfrak{a} / \mathfrak{d}$ games arising in the analysis of uncertainty profiles for the IS-LM and the IS-MP models. We have selected them to illustrate some aspects of the PNE in the $\mathfrak{a} / \mathfrak{d}$ games.

Example 4.1: $\quad$ Take $\mathfrak{M}=$ IS-LM with $\mathcal{E}$ from Example 2.1 and $\mathcal{S}$ from Example 3.1. We will define $\mathcal{A}$ and $\mathcal{D}$ depending on the uncertain scenario we are interested to describe. Consider a situation in which we are interested to know how perturbations affects $Y$ and $r$ when the marginal propensity to consume might be perturbed in an angelic way while the price of goods and taxes might be perturbed in a daemonic way. However, the exogenous government spending might be perturbed in both directions. In consequence we set $\mathcal{A}=$ $\{b, G\}$ and $\mathcal{D}=\{P, G, T\}$. Assume that we do not expect perturbations to be exerted at the same time on more than one component. So, we set $b_{\mathfrak{a}}=b_{\mathfrak{d}}=1$. We are interested to know how perturbations affects $Y$ and $r$. So, we take $u_{\mathfrak{a}}=Y$ and we need to precise the interests of $\mathfrak{d}$ with respect to $r$.

First, we want to catch a "worst-case" situation where the perturbation increases the interest rate. The dictum, "having hight interest rate is bad" is captured by setting $u_{\mathfrak{d}}=r$. In this case, $\mathfrak{d}$ tries to maximize $r$. The so obtained uncertainty profile $\mathcal{U}_{1}=$ $\langle\mathcal{E}, \mathcal{S},\{b, G\},\{P, G, T\}, 1,2, Y, r\rangle$ mimics an uncertain situation asking at the same time for a hight income and a high interest rate. Second, we consider a "best-case" situation. As raising interest rate $r$ has negative effects, we are interested to know what happens when this is not the case. So, $r$ is considered a dis-utility. Raising $r$ is seen as a negative fact. Thus we define $u_{\mathfrak{d}}=-r$ (note that maximizing $-r$ is the same as minimizing $r$ ). These considerations lead to the uncertainty profile $\mathcal{U}_{2}=\langle\mathcal{E}, \mathcal{S},\{b, G\},\{P, G, T\}, 1,2, Y,-r\rangle$. First, let us consider $\mathcal{U}_{1}$ and analyse $\Gamma\left(\mathcal{U}_{1}\right)$. We have $A_{\mathfrak{a}}=\{a \subseteq\{b, G\} \mid \# a=1\}=\{\{b\},\{G\}\}$ and $A_{\mathfrak{d}}=\{d \subseteq\{P, G, T\} \mid \# d=2\}=\{\{P, G\},\{P, T\},\{T, G\}\}$. The utilities are $u_{\mathfrak{a}}=Y$ and $u_{\mathfrak{d}}=r$ which can be tabulated in the usual bi-matrix form. Computing the utilities of $\mathfrak{a}$ and $\mathfrak{d}$ (according to Example 3.3), after applying the perturbation to the selected components, we get, for example $Y(\{b\},\{P, G\}) \approx 1062.96$ and $r(\{b\},\{P, G\}) \approx 7.29$. The remaining results are summarized in the following bi-matrix representation of $\Gamma\left(\mathcal{U}_{1}\right)$.

\begin{tabular}{|c|c|c|c|c|}
\cline { 3 - 5 } \multicolumn{2}{c|}{} & \multicolumn{3}{c|}{$\mathfrak{d}$} \\
\hline \multirow{3}{*}{$\mathfrak{a}$} & $\{b\}$ & $1062.96,7.29$ & $1029.62,6.962$ & $1233.33,22 / 3 \approx 7.33$ \\
& $\{G\}$ & $1066.66,22 / 3 \approx 7.33$ & $1041.66,7.08$ & $1075,5.75$ \\
\hline
\end{tabular}

and $B_{\mathfrak{a}}(\{P, G\})=\{\{G\}\}, B_{\mathfrak{a}}(\{P, T\})=\{\{G\}\}, B_{\mathfrak{a}}(\{T, G\})=\{\{b\}\}$ and, in the case of $\mathfrak{d}$, best responses are $B_{\mathfrak{d}}(\{b\})=\{\{T, G\}\}, B_{\mathfrak{d}}(\{G\})=\{\{P, G\}\}$. Thus $\operatorname{PNE}\left(\Gamma\left(\mathcal{U}_{1}\right)\right)=$ $\{(\{G\},\{P, G\}),(\{b\},\{T, G\})\}$. Observe that $\mathfrak{a}$ and $\mathfrak{d}$ get different rewards in the different PNE. In the case of $\mathcal{U}_{2}$ we find $\operatorname{PNE}\left(\Gamma\left(\mathcal{U}_{2}\right)\right)=\emptyset$. Many other cases might be of interest. In any case, the analyser should transform a perception into an uncertainty profiles.

Example 4.2: Let us continue with the Examples 3.2 and 3.4 of the IS-MP model. We consider the uncertainty profiles where $u_{\mathfrak{a}}=Y$ and analyse the situations in which $\mathfrak{d}$ has opposite interests in the inflation $\pi$, therefore we consider:

$$
\mathcal{U}_{1}=\left\langle\mathcal{E}, \mathcal{S},\{\epsilon, \bar{Y}\},\left\{v, \pi^{*}\right\}, 1,1, Y, \pi\right\rangle, \mathcal{U}_{2}=\left\langle\mathcal{E}, \mathcal{S},\{\epsilon, \bar{Y}\},\left\{v, \pi^{*}\right\}, 1,1, Y,-\pi\right\rangle
$$

and $\operatorname{PNE}\left(\Gamma\left(\mathcal{U}_{1}\right)\right)=\left\{\left(\{\bar{Y}\},\left\{\pi^{*}\right\}\right)\right\}, \operatorname{PNE}\left(\Gamma\left(\mathcal{U}_{2}\right)\right)=\{(\{\bar{Y}\},\{v\})\}$ 
It is well know that although a strategic game might not have a PNE, it always has a Nash equilibrium on mixed strategies (Osborne \& Rubinstein, 1994). A mixed strategies for a player is a probability distribution on its set of actions. Thus in an $\mathfrak{a} / \mathfrak{d}$ game, a mixed strategy profile is a tuple $(\alpha, \beta)$ where $\alpha: A_{\mathfrak{a}} \rightarrow[0,1]$ and $\beta: A_{\mathfrak{d}} \rightarrow[0,1]$ are probability distributions. The utility for player $\mathfrak{p} \in\{\mathfrak{a}, \mathfrak{d}\}$ is defined as $u_{\mathfrak{p}}(\alpha, \beta)=$ $\sum_{(a, d) \in A_{\mathfrak{a}} \times A_{\mathfrak{o}}} \alpha(a) \beta(d) u_{\mathfrak{p}}(a, d)$.

Example 4.3: Consider $\Gamma\left(\mathcal{U}_{1}\right)$ defined in Example 4.1. Taking $0 \leq x, y, z \leq 1$ and $y+$ $z \leq 1$, let $(\alpha, \beta)$ a mixed strategy for $\Gamma\left(\mathcal{U}_{1}\right)$ such that $\alpha=(\alpha(\{b\}), \alpha(\{G\}))=(x, 1-x)$ and $\beta=(\beta(\{P, G\}), \beta(\{P, T\}), \beta(\{T, G\}))=(y, z, 1-y-z)$. Setting $x=1 / 3, y=$ $1 / 4$ and $z=1 / 2$ we get $(\alpha, \beta)=((1 / 3,2 / 3),(1 / 4,1 / 2,1 / 4))$. Utilities are $u_{\mathfrak{a}}(\alpha, \beta)=$ 1067.124 and $u_{\mathfrak{d}}(\alpha, \beta)=6.9195$.

The definition of Nash equilibrium extends to mixed strategies. Following, we adapt to $\mathfrak{a} / \mathfrak{d}$ games the characterization given in (Osborne, 2004).

Property 8: A mixed strategy profile $(\alpha, \beta)$ is a mixed Nash equilibrium in $\Gamma(\mathcal{U})$ if the following two symmetric conditions hold. For all $a \in A_{\mathfrak{a}}$, when $\alpha(a)>0$ we have $u_{\mathfrak{a}}(\alpha, \beta)=u_{\mathfrak{a}}(a, \beta)$, otherwise $u_{\mathfrak{a}}(\alpha, \beta) \geq u_{\mathfrak{a}}(a, \beta)$. For all $d \in A_{\mathfrak{d}}$, when $\beta(d)>0$ we have $u_{\mathfrak{d}}(\alpha, \beta)=u_{\mathfrak{d}}(\alpha, d)$, otherwise $u_{\mathfrak{d}}(\alpha, \beta) \geq u_{\mathfrak{d}}(\alpha, d)$.

Example 4.4: Let us continue with the Example 4.1. The $\mathfrak{a} / \mathfrak{d}$ game corresponding to $\mathcal{U}_{2}=\langle\mathcal{E}, \mathcal{S},\{b, G\},\{P, G, T\}, 1,2, Y,-r\rangle$ has no PNE. Based on property 8 we find that $(\alpha, \beta)=((0.78174,0.21826),(0,0.9293303,0.07066972)$ is a mixed Nash equilibrium. Utilities are $u_{\mathfrak{a}}(\alpha, \beta)=1044.016$ and $u_{\mathfrak{d}}(\alpha, \beta)=-6.98775$.

Games can have more than one Nash equilibrium and get different utilities in those situations. This property does not happen in the case of two players zero-sum games (von Neumann \& Morgenstern, 1944). All the Nash equilibria have the same utility for the first player, this utility is called the value of the game. In a zero-sum $\mathfrak{a} / \mathfrak{d}$ game, $u_{\mathfrak{d}}+u_{\mathfrak{a}}=0$. We model this situation by considering a unique objective function $u$ so that $u_{\mathfrak{a}}=u$ and $u_{\mathfrak{d}}=-u$, we write $\mathcal{U}=\left\langle\mathcal{E}, \mathcal{S}, \mathcal{A}, \mathcal{D}, b_{\mathfrak{a}}, b_{\mathfrak{d}}, u\right\rangle$. The value of $\Gamma(\mathcal{U})$ is denoted by $\nu(\mathcal{U})$ is expressed as $\nu(\mathcal{U})=\min _{\alpha \in \Delta \mathfrak{a}} \max _{\beta \in \Delta_{\mathfrak{o}}} u(\alpha, \beta)=\max _{\beta \in \Delta_{\mathfrak{o}}} \min _{\alpha \in \Delta_{\mathfrak{a}}} u(\alpha, \beta)$. A way to get a zerosum is to consider $(a, d)$ as a perturbation of $(\emptyset, \emptyset)$, then $\mathcal{U}^{\prime}=\left\langle\mathcal{E}, \mathcal{S}, \mathcal{A}, \mathcal{D}, b_{\mathfrak{a}}, b_{\mathfrak{d}}, u\right\rangle$ where $u(a, d)=u_{\mathfrak{a}}(a, d)-\left(u_{\mathfrak{a}}(\emptyset, \emptyset) / u_{\mathfrak{d}}(\emptyset, \emptyset)\right) u_{\mathfrak{d}}(a, d)$.

Example 4.5: Given $\mathcal{U}_{1}$ in Example 4.1, consider $\mathcal{U}=\langle\mathcal{E}, \mathcal{S},\{b, G\},\{P, G, T\}, 1,2, u\rangle$ with $u(a, d)=Y(a, d)-(Y(\emptyset, \emptyset / r(\emptyset, \emptyset)) r(a, d)$. From lemma $1,(Y(\emptyset, \emptyset), r(\emptyset, \emptyset))=$ $(Y, r)=(1100,6)$. As $1100 / 6=550 / 3$ we get $u(a, d)=Y(a, d)-(550 / 3) r(a, d)$. The corresponding zero-sum $\mathfrak{a} / \mathfrak{d}$ game $\Gamma(\mathcal{U})$ is described by the table of $u$ :

\begin{tabular}{|c|c|c|c|c|}
\hline & \multicolumn{3}{|c|}{$\mathfrak{d}$} \\
\hline & & $\{P, G\}$ & $\{P, T\}$ & $\{T, G\}$ \\
\hline $\mathfrak{a}$ & $\begin{array}{l}\{b\} \\
\{G\}\end{array}$ & $\begin{array}{c}-22250 / 81 \approx-274.69 \\
-2500 / 9 \approx-277.77\end{array}$ & $\begin{array}{l}-20000 / 81 \approx-246.91 \\
-4625 / 18 \approx-256.94\end{array}$ & $\begin{aligned}-1000 / 9 & \approx-111.11 \\
125 / 6 & \approx 20.83\end{aligned}$ \\
\hline
\end{tabular}

In this case, there is one PNE at $(\{b\},\{P, G\})$, therefore we know that all NE will provide the same utility and that $\nu(\mathcal{U})=-22250 / 81$. 
Example 4.6: Let us reconsider the Example 4.2. This leads to the uncertainty profile $\mathcal{U}=$ $\left\langle\mathcal{E}, \mathcal{S},\{\epsilon, \bar{Y}\}, \mathcal{D}=\left\{v, \pi^{*}\right\}, 1,1, u\right\rangle$ where $u(a, d)=Y(a, d)-(Y(\emptyset, \emptyset) / \pi(\emptyset, \emptyset)) \pi(a, d)$. According to Example 2.2, $Y(\emptyset, \emptyset)=1306 / 13$ and $\pi(\emptyset, \emptyset)=34 / 13$. In this case $\Gamma(\mathcal{U})$ has a $\operatorname{PNE}\left(\{\bar{Y}\},\left\{\pi^{*}\right\}\right)$ and $\nu(\mathcal{U})=-1534 / 17 \approx-90.23$.

\section{Uncertainty in the IS-LM Model}

We consider the case in which a perturbation is exerted only on the fiscal policy, i.e., $\mathcal{A}=\mathcal{D}=\{G, T\}$. Furthermore, we assume also that $\mathfrak{a}$ and $\mathfrak{d}$ can control just one of the components. We analyse two situations with respect to the utilities. In the first case $\mathfrak{a}$ 's objective is to increase the income as much as possible while $\mathfrak{d}$ tries to increase the interest rate. In the second case we considerer a zero-sum approach taking $u=Y-k r$.

\subsection{A Case of Fiscal Policy under Uncertainty in the IS-LM Model}

Consider the case where $\mathfrak{a}$ and $\mathfrak{d}$ have the capability to act over $T$ and $G$, that is $A_{\mathfrak{a}}=$ $A_{\mathfrak{d}}=\{\{T\},\{G\}\}$ and $u_{\mathfrak{a}}=Y$ and $u_{\mathfrak{d}}=r$ (as it was the case in Example 4.1). We ask if the addition of uncertainty can generate a situation in which no PNE exists. We provide a negative answer. Before stating the result. Let us remind, the definition of dominant strategy equilibrium (DSE) taken from (Osborne \& Rubinstein, 1994). A DSE is a joint action $(a, d)$ such that, for any other joint action $\left(a^{\prime}, d^{\prime}\right) \in A_{\mathfrak{a}} \times A_{\mathfrak{d}}, u_{\mathfrak{a}}\left(a, d^{\prime}\right) \geq u_{\mathfrak{a}}\left(a^{\prime}, d^{\prime}\right)$ and $u_{\mathfrak{d}}\left(a^{\prime}, d\right) \geq u_{\mathfrak{d}}\left(a^{\prime}, d^{\prime}\right)$. As pointed out in (Osborne \& Rubinstein, 1994), in a DSE, the action of every player is a best response independently on which are the actions taken by the other players. Our next result proves the existence of DSE when the perturbation has to be exerted only in one component

Theorem 9: Let $\mathcal{S}$ be a perturbation strength model, for the IS-LM model, such that $\delta_{\mathfrak{p}}(e)=0$ for $e \in\{a, b, c, d, e, f, M, P\}$ and $\mathfrak{p} \in\{\mathfrak{a}, \mathfrak{d}\}$. For the uncertainty profile $\mathcal{U}=$ $\langle\mathcal{E}, \mathcal{S},\{G, T\},\{G, T\}, 1,1, Y, r\rangle$, the following $\Gamma(\mathcal{U})$ has always a DSE:

\begin{tabular}{|c|c|c|c|}
\hline & \multicolumn{2}{|c|}{$\mathfrak{d}$} \\
\hline & & $\{T\}$ & $\{G\}$ \\
\hline \multirow{2}{*}{\multicolumn{2}{|c|}{$T$}} & $u_{\mathfrak{a}}=Y-\frac{f}{g} b\left(\delta_{\mathfrak{a}}(T)+\delta_{\mathfrak{d}}(T)\right)$ & $u_{\mathfrak{a}}=Y+\frac{f}{g}\left(\delta_{\mathfrak{d}}(G)-b \delta_{\mathfrak{a}}(T)\right)$ \\
\hline & & $u_{\mathfrak{d}}=r-\frac{e}{g} b\left(\delta_{\mathfrak{a}}(T)+\delta_{\mathfrak{d}}(T)\right)$ & $u_{\mathfrak{d}}=r+\frac{e}{g}\left(\delta_{\mathfrak{d}}(G)-b \delta_{\mathfrak{a}}(T)\right)$ \\
\hline $\mathfrak{a}$ & \multirow{2}{*}{$G$} & $u_{\mathfrak{a}}=Y+\frac{f}{g}\left(\delta_{\mathfrak{a}}(G)-b \delta_{\mathfrak{d}}(T)\right)$ & $u_{\mathfrak{a}}=Y+\frac{f}{g}\left(\delta_{\mathfrak{a}}(G)+\delta_{\mathfrak{d}}(G)\right)$ \\
\hline & & $u_{\mathfrak{d}}=r+\frac{e}{q}\left(\delta_{\mathfrak{a}}(G)-b \delta_{\mathfrak{d}}(T)\right)$ & $u_{\mathfrak{d}}=r+\frac{e}{q}\left(\delta_{\mathfrak{a}}(G)+\delta_{\mathfrak{d}}(G)\right)$ \\
\hline
\end{tabular}

Proof: Defining $\mu_{\mathfrak{p}, T \rightarrow G}=b \delta_{\mathfrak{p}}(T)+\delta_{\mathfrak{p}}(G)$ it holds that

$$
B_{\mathfrak{p}}(\{T\})=B_{\mathfrak{p}}(\{G\})= \begin{cases}\{G\} & \text { if } \mu_{\mathfrak{p}, T \rightarrow G}>0 \\ \{T, G\} & \text { if } \mu_{\mathfrak{p}, T \rightarrow G}=0 \\ \{T\} & \text { if } \mu_{\mathfrak{p}, T \rightarrow G}<0\end{cases}
$$


Uncertainty in Basic Macroeconomic models

According to the best responses, by case analysis, we find the following PNE structure:

\begin{tabular}{c||c|c|c} 
& $\mu_{\mathfrak{a}, T \rightarrow G}>0$ & $\mu_{\mathfrak{a}, T \rightarrow G}=0$ & $\mu_{\mathfrak{a}, T \rightarrow G}<0$ \\
\hline$\mu_{\mathfrak{d}, T \rightarrow G}>0$ & $\{G, G\}$ & $\{G, T\},\{G, G\}$ & $\{G, T\}$ \\
$\mu_{\mathfrak{d}, T \rightarrow G}=0$ & $\{T, G\}\{G, G\}$ & $\{G, G\}\{G, T\}\{T, G\}\{T, T\}$ & $\{T, T\}\{G, T\}$ \\
$\mu_{\mathfrak{d}, T \rightarrow G}<0$ & $\{T, G\}$ & $\{T, T\}\{T, G\}$ & $\{T, T\}$
\end{tabular}

To prove the existence of a DSE we consider first the case $\mu_{\mathfrak{p}, T \rightarrow G}>0$, for $\mathfrak{p} \in\{\mathfrak{a}, \mathfrak{d}\}$. In such a case, $(\{G\},\{G\})$ is a dominant strategy equilibrium. Other cases are similar.

Consider balanced budgets where the government spends the amount $\delta$ collected by taxation. The strength model $\mathcal{S}_{\text {balanced }}$ verifies $\delta_{\mathfrak{p}}(e)=0$ for $e \in\{a, b, c, d, e, f, M, P\}$ for $\mathfrak{p} \in\{\mathfrak{a}, \mathfrak{d}\}$, as in Theorem 9, moreover and $\delta_{\mathfrak{a}}(T)=\delta_{\mathfrak{d}}(G)=0$ and $\delta_{\mathfrak{a}}(G)=\delta_{\mathfrak{d}}(T)=\delta$. The only PNE of $\Gamma\left(\left\langle\mathcal{E}, \mathcal{S}_{\text {balanced }},\{G, T\},\{G, T\}, 1,1, Y,-r\right\rangle\right)$ is $(\{G\},\{T\})$. This Nash equilibrium corresponds precisely to the case where Government spends the amount $\delta$ collected by taxes and this corresponds to a balanced budget.

\subsection{Direct Control over Income and Interest Rate from $\mathfrak{a}$ and $\mathfrak{d}$}

We consider now the case where $\mathfrak{a}$ is interested in controlling both, $Y$ and $r$, directly.

Theorem 10: Let $\mathcal{S}$ be a perturbation strength model for the IS-LM model such that $\delta_{\mathfrak{p}}(e)=0$ for $e \in\{a, b, c, d, e, f, M, P\}$ and $\mathfrak{p} \in\{\mathfrak{a}, \mathfrak{d}\}$ moreover $\delta_{\mathfrak{a}}(G)=-\delta_{\mathfrak{d}}(G)=$ $\delta_{G} \geq 0$ and $\delta_{\mathfrak{d}}(T)=-\delta_{\mathfrak{a}}(T)=\delta_{T}>0$. Take $\mathcal{U}=\langle\mathcal{E}, \mathcal{S},\{T, G\},\{T, G\}, 1,1, u\rangle$ where $u(a, d)=Y(a, d)-k r(a, d)$. Letting $\delta=b \delta_{T}-\delta_{G}$, the $\Gamma(\mathcal{U})$ game is given by

\begin{tabular}{|c|c|c|c|}
\hline & \multicolumn{2}{|c|}{$\mathfrak{d}$} \\
\hline & & $\{T\}$ & $\{G\}$ \\
\hline \multirow{2}{*}{$\mathfrak{a}$} & $\{T\}$ & $Y-k r$ & $(Y-k r)+(1 / g)(f-k e) \delta$ \\
\hline & $\{G\}$ & $(Y-k r)-(1 / g)(f-k e) \delta$ & $Y-k r$ \\
\hline
\end{tabular}

When $(f-k e) \delta=0$, the four strategy profiles of $\Gamma(\mathcal{U})$ are PNE, otherwise either $(\{T\},\{T\})$ or $(\{G\},\{G\})$ is the unique PNE of $\Gamma(\mathcal{U})$.

Proof: When $(f-k e) \delta=0$ all the pure strategies have the same utility $Y-k r$ and therefore all of them are PNE. When $Y-k r>0$ and $(f-k e) \delta>0$ the only PNE is $(\{T\},\{T\})$. When $Y-k r>0$ and $(f-k e) \delta<0$ the only PNE is $(\{G\},\{G\})$. When $Y-k r<0$ and $(f-k e) \delta>0$ the only PNE is $(\{T\},\{T\})$. When $Y-k r<0$ and $(f-k e) \delta<0$ the only PNE is $(\{G\},\{G\})$. Finally, when $k=Y / r$, we have $u(a, d)=$ $Y(a, d)-(Y / r) r(a, d)$. The $\mathfrak{a} / \mathfrak{d}$ game has $(\{T\},\{T\})$ as PNE in when $u(\{T\},\{G\})>0$. When $u(\{T\},\{G\})<0$ the PNE is $(\{G\},\{G\})$.

\section{Uncertainty in the IS-MP Model}

Now we consider the case where the income $Y$ and the inflation $\pi$ become uncertain due to perturbations in $\left\{\bar{Y}, \pi^{*}\right\}$. We analyse the case where a benevolent $\mathfrak{a}$ tries to keep the income as high as possible, $u_{\mathfrak{a}}=Y$. As in Example 4.2, we consider different views of $\mathfrak{d}$ in relation to $r$. To model the case where $\mathfrak{d}$ tries to maximize the inflation, we take $u_{\mathfrak{d}}=\pi$. 
To model the case where $\pi$ is a dis-utility $\mathfrak{d}$ tries to minimize $\pi$, we take $u_{\mathfrak{d}}=-\pi$. The following theorem consider both cases. The proof follows preceding techniques.

Theorem 11: Let $S$ be a perturbation strength model for the IS-MP model with $\delta_{\mathfrak{a}}(e)=$ $\delta_{\mathfrak{d}}(e)=0$, for any $e \in \mathcal{E} \backslash\left\{\bar{Y}, \pi^{*}\right\}$ and $\delta_{\mathfrak{a}}(\bar{Y})>0$ and $\delta_{\mathfrak{d}}\left(\pi^{*}\right)>0$. Consider and uncertainty profile $\mathcal{U}=\left\langle\mathcal{E}, \mathcal{S},\left\{\bar{Y}, \pi^{*}\right\},\left\{\bar{Y}, \pi^{*}\right\}, 1,1, Y, u_{\mathfrak{d}}\right\rangle$. When $u_{\mathfrak{d}}=\pi$ the $\mathfrak{a} / \mathfrak{d}$ game is

\begin{tabular}{|c|c|c|c|}
\cline { 3 - 4 } \multicolumn{2}{c|}{} & \multicolumn{2}{|c|}{$\mathfrak{d}$} \\
\cline { 3 - 4 } \multicolumn{2}{c|}{} & $\{\bar{Y}\}$ & $\left\{\pi^{*}\right\}$ \\
\hline \multirow{2}{*}{$\mathfrak{a}\}$} & $Y+\delta_{\mathfrak{a}}(\bar{Y})+\delta_{\mathfrak{d}}(\bar{Y}), \pi$ & $Y+\delta_{\mathfrak{a}}(\bar{Y}), \pi+\delta_{\mathfrak{d}}\left(\pi^{*}\right)$ \\
\cline { 3 - 4 } & $\left\{\pi^{*}\right\}$ & $Y+\delta_{\mathfrak{d}}(\bar{Y}), \pi+\delta_{\mathfrak{a}}\left(\pi^{*}\right)$ & $Y, \pi+\delta_{\mathfrak{a}}\left(\pi^{*}\right)+\delta_{\mathfrak{d}}\left(\pi^{*}\right)$ \\
\hline
\end{tabular}

$\{\bar{Y}\}$ is the dominant strategy for $\mathfrak{a}$ and $\left\{\pi^{*}\right\}$ is the dominant strategy for $\mathfrak{d}$. The unique PNE is $\left(\{\bar{Y}\},\left\{\pi^{*}\right\}\right)$ with $Y\left(\{\bar{Y}\},\left\{\pi^{*}\right\}\right)=Y+\delta_{\mathfrak{d}}(\bar{Y})$ and $\pi\left(\{\bar{Y}\},\left\{\pi^{*}\right\}\right)=\pi+\delta_{\mathfrak{a}}\left(\pi^{*}\right)$. When $u_{\mathfrak{d}}=-\pi$ the game is

\begin{tabular}{|c|c|c|c|}
\hline & \multicolumn{2}{|c|}{$\mathfrak{d}$} \\
\hline & & $\{\bar{Y}\}$ & $\left\{\pi^{*}\right\}$ \\
\hline \multirow{2}{*}{$\mathfrak{a}$} & $\{\bar{Y}\}$ & $Y+\delta_{\mathfrak{a}}(\bar{Y})+\delta_{\mathfrak{d}}(\bar{Y}),-\pi$ & $Y+\delta_{\mathfrak{a}}(\bar{Y}),-\pi+\delta_{\mathfrak{d}}\left(\pi^{*}\right)$ \\
\hline & $\left\{\pi^{*}\right\}$ & $Y+\delta_{\mathfrak{d}}(\bar{Y}),-\pi+\delta_{\mathfrak{a}}\left(\pi^{*}\right)$ & $Y,-\pi+\delta_{\mathfrak{a}}\left(\pi^{*}\right)+\delta_{\mathfrak{d}}\left(\pi^{*}\right)$ \\
\hline
\end{tabular}

The dominant strategy for both $\mathfrak{a}$ and $\mathfrak{d}$ is $\{\bar{Y}\}$ and the only PNE is $(\{\bar{Y}\},\{\bar{Y}\})$. In this case $Y(\{\bar{Y}\},\{\bar{Y}\})=Y+\delta_{\mathfrak{a}}(\bar{Y})+\delta_{\mathfrak{d}}(\bar{Y})$ and $\pi(\{\bar{Y}\},\{\bar{Y}\})=\pi$.

We conclude our study considering a subclass of zero-sum $\mathfrak{a} / \mathfrak{d}$ games generalizing the conditions of the uncertainty profiles considered in Example 4.2.

Theorem 12: Let $S$ be a perturbation strength model for the IS-MP model such that $\delta_{\mathfrak{a}}(e)=\delta_{\mathfrak{d}}(e)=0$, for any $e \in \mathcal{P}$. Let $\mathcal{U}=\langle\mathcal{E}, \mathcal{S}, \mathcal{A}, \mathcal{D}, 1,1, u\rangle$ where the utility is $u(a, d)=Y(a, d)-(Y(\{\emptyset\},\{\emptyset\}) / \pi(\{\emptyset\},\{\emptyset\})) \pi(a, d)$. In $\Gamma(\mathcal{U})$, when $\delta_{\mathfrak{p}}(e)>0$, for $\mathfrak{p} \in$ $\{\mathfrak{a}, \mathfrak{d}\}$ and $e \in\left\{\bar{Y}, \pi^{*}\right\},\{\bar{Y}\}$ is the dominant strategy for $\mathfrak{a}$ and $\left\{\pi^{*}\right\}$ is the dominant strategy for $\mathfrak{d}$. Furthermore, $\left(\{\bar{Y}\},\left\{\pi^{*}\right\}\right)$ is the unique PNE.

\section{Conclusions and Further Developments}

We have shown how to adapt the $\mathfrak{a} / \mathfrak{d}$-framework provided in (Gabarro et al., 2014) to analyse uncertainty in the IS-LM and the IS-MP models. In both cases, we have studied different possible cases of uncertainty through the set of Nash equilibria showing the applicability of the framework.

Our approach can be adapted to analyse uncertainty in other financial settings. We do that considering uncertainty in the price $f$ of a call option over a stock $S$. We use the one step binomial tree model (Hull, 1989) having the following components and values:

\begin{tabular}{||c|c|c|c|c|c||}
\hline Stock price & $S=20$ & Time period & $T=0.25$ & Up jump & $u=1.1$ \\
Strike price & $X=21$ & Risk-free rate & $r=0.12$ & Down jump & $d=0.6$ \\
\hline
\end{tabular}


Let us take $\mathcal{E}=\{r, u, d\}$ and $\mathcal{S}$ such that $\delta_{\mathfrak{a}}(r)=-0.05, \delta_{\mathfrak{a}}(u)=0.4, \delta_{\mathfrak{a}}(d)=0$ and $\delta_{\mathfrak{d}}(r)=0.10, \delta_{\mathfrak{d}}(u)=0, \delta_{\mathfrak{d}}(d)=0.3$. Let $\mathcal{U}=\langle\mathcal{E}, \mathcal{S},\{\{u\},\{r\}\},\{\{d\},\{r\}\}, 1,1, f\rangle$ be an uncertainty profile for a call option. The corresponding $\mathfrak{a} / \mathfrak{d}$ game is:

\begin{tabular}{|c|c|c|c|}
\cline { 3 - 4 } \multicolumn{2}{c|}{} & \multicolumn{2}{c|}{$\mathfrak{d}$} \\
\cline { 3 - 4 } $\mathfrak{*} \mathfrak{a}$ & $\{u\}$ & 1.898985 & 4.321089 \\
\cline { 3 - 4 } & $\{r\}$ & 0.5780649 & 0.8499314 \\
\hline
\end{tabular}

The only PNE is $(\{u\},\{d\})$ with $\nu(\mathcal{U})=1.898985$. Therefore, in the uncertain situation described by $\mathcal{U}$ perhaps the price of the call should be 1.898985 rather than 0.835465 .

It is important to know the possibilities and limits of the uncertainty profiles in macroeconomics. As pointed in (Durlauf, 2012), a way to decide with no probabilities is to guard against really bad cases. In such a setting regret analysis provides a way to analyse such cases. Analysing the connection between $\mathfrak{a} / \mathfrak{d}$ and regret analysis would be interesting. In (Nordhaus, 2013), different scenarios are developed in order to analyse different possibilities in relation to climate change.

It is also important to know the possibilities, limits and weaknesses of the $\mathfrak{a} / \mathfrak{d}$ approach. We have considered uncertainty profiles $\mathcal{U}=\left\langle\mathcal{E}, \mathcal{S}, \mathcal{A}, \mathcal{D}, b_{\mathfrak{a}}, b_{\mathfrak{d}}, u_{\mathfrak{a}}, u_{\mathfrak{d}}\right\rangle$ where the spreads $b_{\mathfrak{a}}, b_{\mathfrak{d}}$ are fixed independently of the other parameters in $\mathcal{U}$. It is interesting to consider the case where the spreads are related to other parameters, for instance $b_{\mathfrak{a}}=\left\lfloor\frac{1}{2} \# \mathcal{A}\right\rfloor$ or $b_{\mathfrak{d}}=\left\lfloor\frac{2}{3} \# \mathcal{A}\right\rfloor$. Moreover, any $\mathcal{U}$ is a tuple having many parameters to be determined, the danger of over parametrization exists (Hull, 2010).

Finally, in this paper we have consider only short-time models. In (Castro et al., 2015) a time dimension of the $\mathfrak{a} / \mathfrak{d}$ approach was integrated into the frame of stochastic automata. Perhaps this approach, based on stochastic automata, could be adapted to deal with the uncertainty along the time of some economic models.

\section{Acknowledgements}

We thanks the referees for their fruitful suggestions. J. Gabarro and M. Serna are partially supported by funds from the Spanish Ministry for Economy and Competitiveness (MINECO) and the European Union (FEDER funds) under grant TIN2013-46181-C2-1R (COMMAS) and from AGAUR, Generalitat de Catalunya under grant SGR 2014:1034 (ALBCOM).

\section{References}

Arratia, A. (2014). Computational Finance, An Introductory Course with R. Atlantis Press.

Baldani, J., Bradfield, J., \& Turner, R. (2007). Mathematical Economics, 2nd edition. Thomson.

Castro, J., Gabarro, J., Serna, M. J., \& Stewart, A. (2015). The robustness of periodic orchestrations in uncertain evolving environments. In ECSQARU 2015, Compiègne, France, July 15-17, 2015. Proceedings, vol. 9161 of Lecture Notes in Computer Science, (pp. 129-140). Springer. 
Durlauf, S. (2012). Model uncertainty and empirical policy analysis in Economics: A selective review. Advances in Austrian Economic (Experts and Epistemic Monopolies), $17,203-226$.

ECB (2015). Monetary Policy.

https://www. ecb. europa.eu/mopo/intro.

Gabarro, J., Serna, M., \& Stewart, A. (2014). Analysing web-orchestrations under stress using uncertainty profiles. The Computer Journal, 57(11), 1591-1615.

Hicks, J. (1937). Mr Keynes and the "Classics"; a suggested interpretation. Econometrica, 5(2), 147-157.

Hicks, J. (1980-1981). IS-LM: An explanation. Journal of Post Keynesian Economics, 3, 139-154.

Hull, J. (1989). Options, Futures, and Other Derivatives. Prentica Hall, third ed.

Hull, J. (2010). Risk Management and Financial Institutions. Pearson, second ed.

Knight, F. (1921). Risk, Uncertainty and Profit. Houghton Mifflin.

URL http: / / www . econlib.org/library/Knight/knRUP. html

Krugman, P. (2011). IS-LMentary.

http: //krugman.blogs.nytimes.com/2011/10/09/is-lmentary. International New York Times blog.

Mankiw, G. (2013). Macroeconomics, 8th edition. Worth Publishers.

Nordhaus, W. (2013). The Climate Casino. Yale University Press.

Osborne, M. (2004). An Introduction to Game Theory. New York and Oxford: Oxford University Press.

Osborne, M., \& Rubinstein, A. (1994). A Course on Game Theory. The MIT Press.

Piketty, T. (2014). Capital in the Twenty-First Century. Harvard University Press.

Romer, D. (2000). Keynesian macroeconomics without the LM curve. Journal of Economic Perspectives, 14(2), 149-169.

Taylor, J. (1993). Discretion versus policy rules in practice. Carnegie-Rochester Conference Series on Public Policy, 39, 195 - 214.

von Neumann, J., \& Morgenstern, O. (1944). Theory of Games and Economic Behavior. Princeton and Oxford: Princeton University Press. 